\title{
STEADY STATES FOR A FRAGMENTATION EQUATION WITH SIZE DIFFUSION
}

\author{
PHILIPPE LAURENÇOT \\ Mathématiques pour l'Industrie et la Physique, CNRS UMR 5640 \\ Université Paul Sabatier - Toulouse 3 \\ 118 route de Narbonne \\ F-31062 Toulouse Cedex 4, France \\ E-mail: laurenco@mip.ups-tlse.fr
}

\begin{abstract}
The existence of a one-parameter family of stationary solutions to a fragmentation equation with size diffusion is established. The proof combines a fixed point argument and compactness techniques.
\end{abstract}

1. Introduction. We study the existence and uniqueness of stationary solutions to a model of particle growth in which the size of the particles is altered by diffusion and fragmentation. A typical situation where there is an interplay between these two mechanisms is the growth of ice crystals: indeed, ice crystals grow or shrink in a way which looks like diffusion and are subjected to stresses which could lead to fragmentation [6]. An interesting feature of such a model is that diffusion and fragmentation have somehow opposite effects on the size distribution of the particles: while the fragmentation mechanism "moves" the size distribution towards small sizes, the diffusion mechanism "pushes" the size distribution towards large sizes. Owing to this competition, one might expect a balance to occur between these two effects leading to stationary size distributions. The existence of steady states has been observed in [6] in a particular case and the aim of this paper is to identify a class of data for which steady states exist.

From a mathematical viewpoint, the fragmentation equation with size diffusion describes the evolution of the size distribution function $f(t, x) \geq 0$ of particles of size $x \in(0, \infty)$ at time $t \geq 0$ and reads

$$
\partial_{t} f(t, x)-D \partial_{x}^{2} f(t, x)=-a(x) f(t, x)+\int_{x}^{\infty} a\left(x_{*}\right) b\left(x, x_{*}\right) f\left(t, x_{*}\right) d x_{*} .
$$

2000 Mathematics Subject Classification: Primary 45J05.

Key words and phrases: fragmentation, diffusion, stationary solution.

The paper is in final form and no version of it will be published elsewhere. 
Here, $D>0$ is the diffusion coefficient which is assumed to be a positive constant, $a$ is the fragmentation rate and $b$ the daughter size distribution. In the absence of diffusion, the equation (1) is the well-known fragmentation equation with multiple fragmentation $[7,11]$ which has been studied extensively. We refer to $[4,7,9,12]$ (and the references therein) for the computation of explicit solutions and to $[1,3,5,7,8,10,11]$ (and the references therein) for analytical studies relying on either a deterministic or a stochastic approach. When size diffusion is taken into account, the solution to (1) has been computed explicitly in [6] when $a(x)=x, b\left(x, x_{*}\right)=2$, the equation (1) being supplemented by the homogeneous Dirichlet boundary condition $f(t, 0)=0$ and the requirement that $f$ decays to zero at infinity. These boundary conditions and the choice of $b$ ensure that there is no exchange of matter between the system of particles and the outer medium, that is,

$$
\int_{0}^{\infty} x f(t, x) d x=\text { const. }
$$

through time evolution provided this quantity is initially finite. In addition, a oneparameter family of stationary solutions is exhibited in that case, and our purpose is to extend this result to a larger class of data $a$ and $b$.

We thus consider the boundary-value problem

$$
\begin{aligned}
-\partial_{x}^{2} f(x) & =-a(x) f(x)+\int_{x}^{\infty} a\left(x_{*}\right) b\left(x, x_{*}\right) f\left(x_{*}\right) d x_{*}, \quad x \in(0, \infty), \\
f(0) & =0
\end{aligned}
$$

where we have taken $D=1$ for simplicity. We assume that the fragmentation rate $a$ and the daughter distribution function $b$ fulfil the following conditions:

$$
\begin{gathered}
a(x)=x^{\alpha}, \quad x \in(0, \infty), \quad \alpha \geq 0, \\
b \text { is measurable and } b>0 \text { a.e., } \\
\int_{0}^{x} x_{*} b\left(x_{*}, x\right) d x_{*}=x, \quad x \in(0, \infty),
\end{gathered}
$$

and, for each $\beta \geq 0$, there is a positive constant $B_{\beta}$ such that

$$
\int_{0}^{x} x_{*}^{\beta} b\left(x_{*}, x\right) d x_{*} \leq B_{\beta} x^{\beta}, \quad x \in(0, \infty),
$$

with the additional requirement that

$$
B_{\beta}<1 \text { when } \beta>1 .
$$

A typical example of daughter distribution function $b$ satisfying (6)-(9) is

$$
b\left(x_{*}, x\right)=(\nu+2) x_{*}^{\nu} x^{-\nu-1}, \quad 0<x_{*}<x,
$$

for $\nu>-1\left(\right.$ with $\left.B_{\beta}=(\nu+2) /(\beta+\nu+1)\right)$ [9]. Observe that the case studied in [6] corresponds to the choice $\alpha=1$ in (6) and $\nu=0$ in (10).

We may now state our main result.

Theorem 1. Assume that $a$ and $b$ fulfil the conditions (6)-(9). Given $\varrho \in[0, \infty)$, there is a unique non-negative solution

$$
f_{\varrho} \in W_{0}^{1,1}(0, \infty) \cap W^{2,1}(0, \infty) \cap L^{1}\left(0, \infty ; x^{1+\alpha} d x\right)
$$


to (3), (4) satisfying

$$
\int_{0}^{\infty} x f_{\varrho}(x) d x=\varrho .
$$

In addition, $f_{\varrho} \in L^{1}\left(0, \infty ; x^{\beta} d x\right)$ for each $\beta \geq 0$.

Since (3), (4) is a linear boundary-value problem, we obviously have $f_{\varrho}=\varrho f_{1}$ for $\varrho \geq 0$. We will thus only consider the case $\varrho=1$ in the sequel.

Observe that, under the assumptions (6)-(9), the boundary-value problem (3), (4) has a solution with a fast decay at infinity. Such a property is expected since the assumptions on $a$ and $b$ are somehow reminiscent of the strong fragmentation assumption which is used in the study of coagulation-fragmentation equations with binary fragmentation $[2,5]$. More precisely, under the strong fragmentation assumption, it is known that the $L^{1}\left(0, \infty ; x^{\beta} d x\right)$-norm of solutions to coagulation-fragmentation equations becomes instantaneously finite for positive times for every $\beta>1[2,5]$.

REMARK 2. Let us point out that the conservation of matter (2) discussed previously may fail for the fragmentation equation (1) without diffusion $(D=0)[9]$. This phenomenon is the so-called "shattering" transition and corresponds to the appearance of dust (i.e. particles of size zero). It however only occurs when $a$ is singular for $x=0$ (typically $a(x)=x^{\alpha}$ with $\left.\alpha<0\right)$ and is thus excluded from our analysis. We refer to $[1,4,7,8,9]$ for a more detailed account on the "shattering" transition.

Owing to the unboundedness of $a$ and $b$, the proof of Theorem 1 is split in two parts: we first consider a "truncated" boundary-value problem on a bounded interval $(0, N), N \geq 1$, for which we establish an existence result by the Schauder fixed point theorem. The second step is to deduce from (8) and (9) a bound in $L^{1}\left(0, N ; x^{\beta} d x\right)$ which is independent on $N$. A compactness argument then allows us to complete the existence part of Theorem 1 . The uniqueness part of Theorem 1 relies on the contractivity properties in $L^{1}(0, \infty ; x d x)$ of the fragmentation and diffusive terms.

2. A truncated boundary-value problem. Let $N \geq 1$ be a fixed integer. In this section, we use the Schauder fixed point theorem to show the existence of a non-negative solution $f$ to

$$
\begin{aligned}
& -\partial_{x}^{2} f(x)=-a(x) f(x)+\int_{x}^{N} a\left(x_{*}\right) b\left(x, x_{*}\right) f\left(x_{*}\right) d x_{*}, \quad x \in(0, N), \\
& f(0)=N \partial_{x} f(N)-f(N)=0,
\end{aligned}
$$

such that

$$
\int_{0}^{N} x f(x) d x=1 .
$$

Proposition 3. There is a unique non-negative solution $f \in W^{2,1}(0, N)$ to (12), (13) which satisfies (14).

Proof. (a) Uniqueness. Let $f$ and $\hat{f}$ be two non-negative solutions in $W^{2,1}(0, N)$ to (12), (13) which fulfil (14), and put $F=f-\hat{f}$. Then $F \in W^{2,1}(0, N)$ is also a solution to (12), 
(13) and satisfies

$$
\int_{0}^{N} x F(x) d x=0
$$

We put $\sigma(x)=\operatorname{sign}(F(x)), x \in(0, N)$. On the one hand, we infer from the Kato inequality and (13) that

$$
-\int_{0}^{N} x \sigma(x) \partial_{x}^{2} F(x) d x \leq-N \sigma(N) \partial_{x} F(N)+|F(N)|-|F(0)|=0 .
$$

On the other hand, (7) and the Fubini theorem yield

$$
\begin{aligned}
& \int_{0}^{N} x \sigma(x)\left(-a(x) F(x)+\int_{x}^{N} a\left(x_{*}\right) b\left(x, x_{*}\right) F\left(x_{*}\right) d x_{*}\right) d x \\
= & -\int_{0}^{N} x a(x)|F(x)| d x+\int_{0}^{N} a(x) F(x) \int_{0}^{x} x_{*} b\left(x_{*}, x\right) \sigma\left(x_{*}\right) d x_{*} d x \\
= & -\int_{0}^{N} a(x)|F(x)| \int_{0}^{x} x_{*} b\left(x_{*}, x\right)\left(1-\sigma(x) \sigma\left(x_{*}\right)\right) d x_{*} d x .
\end{aligned}
$$

We now multiply (12) for $F$ by $x \sigma(x)$, integrate over $(0, N)$ and use the previous two inequalities to conclude that

$$
\int_{0}^{N} a(x)|F(x)| \int_{0}^{x} x_{*} b\left(x_{*}, x\right)\left(1-\sigma(x) \sigma\left(x_{*}\right)\right) d x_{*} d x \leq 0 .
$$

Since $\sigma(x) \sigma\left(x_{*}\right) \leq 1$, the positivity of $a$ and $b$ entail that

$$
|F(x)|\left(1-\sigma(x) \sigma\left(x_{*}\right)\right) \mathbf{1}_{(0, x)}\left(x_{*}\right)=0 \text { a.e. in }(0, N) \times(0, N) .
$$

Introducing

$$
\begin{aligned}
& \mathcal{P}_{k}=\left\{x \in(0, N), \quad F(x) \geq \frac{1}{k}\right\} \quad \text { for } \quad k \geq 1 \text { and } \mathcal{P}=\bigcup_{k \geq 1} \mathcal{P}_{k}, \\
& \mathcal{N}_{k}=\left\{x \in(0, N), \quad F(x) \leq-\frac{1}{k}\right\} \quad \text { for } \quad k \geq 1 \text { and } \mathcal{N}=\bigcup_{k \geq 1} \mathcal{N}_{k},
\end{aligned}
$$

we deduce from (16) that

$$
\begin{aligned}
\frac{2}{k}\left|\mathcal{P}_{k}\right|\left|\mathcal{N}_{k}\right| & =\frac{2}{k} \int_{0}^{N} \int_{0}^{x} \mathbf{1}_{\mathcal{P}_{k}}(x) \mathbf{1}_{\mathcal{N}_{k}}\left(x_{*}\right) d x_{*} d x \\
& +\frac{2}{k} \int_{0}^{N} \int_{0}^{x_{*}} \mathbf{1}_{\mathcal{P}_{k}}(x) \mathbf{1}_{\mathcal{N}_{k}}\left(x_{*}\right) d x_{*} d x \\
& =\frac{1}{k} \int_{0}^{N} \int_{0}^{x} \mathbf{1}_{\mathcal{P}_{k}}(x) \mathbf{1}_{\mathcal{N}_{k}}\left(x_{*}\right)\left(1-\sigma(x) \sigma\left(x_{*}\right)\right) d x_{*} d x \\
& +\frac{1}{k} \int_{0}^{N} \int_{0}^{x_{*}} \mathbf{1}_{\mathcal{P}_{k}}(x) \mathbf{1}_{\mathcal{N}_{k}}\left(x_{*}\right)\left(1-\sigma(x) \sigma\left(x_{*}\right)\right) d x_{*} d x \\
& \leq \int_{0}^{N} \int_{0}^{x}|F(x)|\left(1-\sigma(x) \sigma\left(x_{*}\right)\right) d x_{*} d x \\
& +\int_{0}^{N} \int_{0}^{x_{*}}\left|F\left(x_{*}\right)\right|\left(1-\sigma(x) \sigma\left(x_{*}\right)\right) d x_{*} d x \\
& \leq 0
\end{aligned}
$$


Consequently, $\left|\mathcal{P}_{k}\right|\left|\mathcal{N}_{k}\right|=0$ for every $k \geq 1$ and letting $k \rightarrow \infty$ entail that $|\mathcal{P}||\mathcal{N}|=0$. We have thus shown that either $F \geq 0$ a.e. or $F \leq 0$ a.e., which, together with (15), implies that $F=0$ and completes the uniqueness proof.

(b) Existence. We put $\omega=N^{\alpha / 2}=\|a\|_{L^{\infty}(0, N)}^{1 / 2}$. For $g \in L^{1}(0, N)$, we denote by $u_{g}$ the unique solution in $W^{2,1}(0, N)$ to the boundary-value problem

$$
\begin{aligned}
& -\partial_{x}^{2} u_{g}+\omega^{2} u_{g}=g, \quad x \in(0, N), \\
& u_{g}(0)=N \partial_{x} u_{g}(N)-u_{g}(N)=0 .
\end{aligned}
$$

We recall that $u_{g}$ is given by

$$
u_{g}(x)=\left(\lambda-\int_{0}^{x} e^{-\omega x_{*}} g\left(x_{*}\right) d x_{*}\right) \frac{e^{\omega x}}{2 \omega}-\left(\lambda-\int_{0}^{x} e^{\omega x_{*}} g\left(x_{*}\right) d x_{*}\right) \frac{e^{-\omega x}}{2 \omega}
$$

with

$$
\begin{aligned}
& \lambda=\vartheta \int_{0}^{N} e^{-\omega x_{*}} g\left(x_{*}\right) d x_{*}+(1-\vartheta) \int_{0}^{N} e^{\omega x_{*}} g\left(x_{*}\right) d x_{*}, \\
& \vartheta=\frac{N \omega-1}{N \omega-1+(N \omega+1) e^{-2 \omega N}} .
\end{aligned}
$$

In particular, there is a constant $\Gamma_{N}$ depending on $N$ such that

$$
\left\|u_{g}\right\|_{W^{1, \infty}(0, N)} \leq \Gamma_{N}\|g\|_{L^{1}(0, N)} .
$$

Next, for $f \in L^{1}(0, N)$, we define

$$
\mathcal{L}(f)(x)=\left(\omega^{2}-a(x)\right) f(x)+\int_{x}^{N} a\left(x_{*}\right) b\left(x, x_{*}\right) f\left(x_{*}\right) d x_{*}, \quad x \in(0, N) .
$$

Owing to the choice of $\omega$, we have $\mathcal{L}(f) \geq 0$ whenever $f \geq 0$ and straightforward computations, (7) and (8) yield

$$
\begin{gathered}
\int_{0}^{N} x \mathcal{L}(f)(x) d x=\omega^{2} \int_{0}^{N} x f(x) d x, \\
\|\mathcal{L}(f)\|_{L^{1}(0, N)} \leq \omega^{2}\left(2+B_{0}\right)\|f\|_{L^{1}(0, N)} .
\end{gathered}
$$

We finally introduce the set

$$
\mathcal{C}=\left\{f \in L^{1}(0, N), \quad f \geq 0, \quad \int_{0}^{N} x f(x) d x=1, \quad\|f\|_{L^{1}(0, N)} \leq R_{\star}\right\},
$$

with

$$
R_{\star}=N \omega^{2}+\frac{N}{2}\left(3+\omega^{2}\right),
$$

which is a bounded closed and convex subset of $L^{1}(0, N)$. For $f \in \mathcal{C}$, we define $\Lambda(f)=$ $u_{\mathcal{L}(f)}$ and first check that $\Lambda(f)$ also belongs to $\mathcal{C}$. Indeed, it is clear that $u_{\mathcal{L}(f)} \in$ $W^{2,1}(0, N) \subset L^{1}(0, N)$. Next, since $f \geq 0$, we have also $\mathcal{L}(f) \geq 0$ and the maximum principle implies that $u_{\mathcal{L}(f)} \geq 0$. It also readily follows from (17), (18) and (20) that

$$
\int_{0}^{N} x u_{\mathcal{L}(f)}(x) d x=1 .
$$


Now, we multiply (17) for $\Lambda(f)$ by $x^{3}$, integrate over $(0, N)$ and use $(7),(18)$ and (22) to obtain

$$
\begin{gathered}
-N^{3} \partial_{x} \Lambda(f)(N)+3 N^{2} \Lambda(f)(N)-6 \int_{0}^{N} x \Lambda(f)(x) d x+\omega^{2} \int_{0}^{N} x^{3} \Lambda(f)(x) d x \\
=\int_{0}^{N} x^{3}\left(\omega^{2}-a(x)\right) f(x) d x+\int_{0}^{N} a\left(x_{*}\right) f\left(x_{*}\right) \int_{0}^{x_{*}} x^{3} b\left(x, x_{*}\right) d x d x_{*} \\
2 N^{2} \Lambda(f)(N)-6 \leq \omega^{2} \int_{0}^{N} x^{3} f(x) d x+N^{2} \int_{0}^{N} a\left(x_{*}\right) f\left(x_{*}\right) x_{*} d x_{*} \\
\leq N^{2} \omega^{2} \int_{0}^{N} x f(x) d x+N^{2} \omega^{2} \int_{0}^{N} x f(x) d x \\
\leq 2 N^{2} \omega^{2},
\end{gathered}
$$

whence

$$
\Lambda(f)(N) \leq 3+\omega^{2} .
$$

We then multiply (17) for $\Lambda(f)$ by $x^{2}$, integrate over $(0, N)$ and use $(18)$ and the nonnegativity of $f, a$ and $b$ to obtain

$$
\begin{gathered}
N \Lambda(f)(N)-2 \int_{0}^{N} \Lambda(f)(x) d x+\omega^{2} \int_{0}^{N} x^{2} \Lambda(f)(x) d x \geq-\int_{0}^{N} x^{2} a(x) f(x) d x \\
N \Lambda(f)(N)+\omega^{2} \int_{0}^{N} x^{2} \Lambda(f)(x) d x+\int_{0}^{N} x^{2} a(x) f(x) d x \geq 2 \int_{0}^{N} \Lambda(f)(x) d x,
\end{gathered}
$$

whence, thanks to $(22)$ and (23),

$$
2 \int_{0}^{N} \Lambda(f)(x) d x \leq N\left(3+\omega^{2}\right)+N \omega^{2}+N \omega^{2}=2 R_{\star} .
$$

Consequently, $\Lambda$ maps $\mathcal{C}$ into itself. In addition, we infer from (19) and (21) that, for $f \in \mathcal{C}$ and $\hat{f} \in \mathcal{C}$, we have

$\left\|u_{\mathcal{L}(f)}-u_{\mathcal{L}(\hat{f})}\right\|_{L^{1}(0, N)}=\left\|u_{\mathcal{L}(f)-\mathcal{L}(\hat{f})}\right\|_{L^{1}(0, N)}=\left\|u_{\mathcal{L}(f-\hat{f})}\right\|_{L^{1}(0, N)} \leq C(N)\|f-\hat{f}\|_{L^{1}(0, N)}$.

Therefore, $\Lambda$ maps continuously $\mathcal{C}$ into itself and $\Lambda(\mathcal{C})$ is compact in $L^{1}(0, N)$ by the compactness of the embedding of $W^{1, \infty}(0, N)$ in $L^{1}(0, N)$. We are then in a position to employ the Schauder fixed point theorem and conclude that there exists $f \in \mathcal{C}$ such that $\Lambda(f)=f$. In other words, $f$ satisfies (13) and (14), and

$$
-\partial_{x}^{2} f(x)+\omega^{2} f(x)=\left(\omega^{2}-a(x)\right) f(x)+\int_{x}^{N} a\left(x_{*}\right) b\left(x, x_{*}\right) f\left(x_{*}\right) d x_{*}
$$

for $x \in(0, N)$, i.e. $f$ satisfies $(12)$.

3. Proof of Theorem 1. For $N \geq 1$, we denote by $f_{N}$ the solution to (12), (13) satisfying (14) given by Proposition 3 , and we still denote by $f_{N}$ its extension by zero to $(0, \infty)$. In particular, we have

$$
f_{N} \geq 0, \quad \int_{0}^{\infty} x f_{N}(x) d x=1
$$


Lemma 4. For each $\beta \geq 3$, there is a constant $C_{\beta}$ depending only on $\alpha$ and $\beta$ such that

$$
N^{\beta-1} f_{N}(N)+\int_{0}^{N} x^{\alpha+\beta} f_{N}(x) d x \leq C_{\beta} .
$$

There is a positive constant $C_{0}$ depending only on $\alpha$ such that

$$
\int_{0}^{N} f_{N}(x) d x \leq C_{0} .
$$

Proof. We multiply (12) by $x^{\beta}$, integrate over $(0, N)$ and use (8), (9) and (13) to obtain

$$
\begin{aligned}
& -N^{\beta} \partial_{x} f_{N}(N)+\beta N^{\beta-1} f_{N}(N)-\beta(\beta-1) \int_{0}^{N} x^{\beta-2} f_{N}(x) d x \\
= & \int_{0}^{N} a(x) f_{N}(x) \int_{0}^{x} x_{*}^{\beta} b\left(x_{*}, x\right) d x_{*} d x-\int_{0}^{N} a(x) x^{\beta} f_{N}(x) d x
\end{aligned}
$$

$$
\begin{aligned}
& (\beta-1) N^{\beta-1} f_{N}(N)-\beta(\beta-1) \int_{0}^{N} x^{\beta-2} f_{N}(x) d x \leq\left(B_{\beta}-1\right) \int_{0}^{N} a(x) x^{\beta} f_{N}(x) d x, \\
& (27) \quad N^{\beta-1} f_{N}(N)+\left(1-B_{\beta}\right) \int_{0}^{N} x^{\alpha+\beta} f_{N}(x) d x \leq \beta(\beta-1) \int_{0}^{N} x^{\beta-2} f_{N}(x) d x .
\end{aligned}
$$

Since $\beta \geq 3$, the Young inequality and (24) ensure that there is a positive constant $C_{\beta}$ depending on $\alpha$ and $\beta$ such that

$$
\begin{aligned}
\int_{0}^{N} x^{\beta-2} f_{N}(x) d x & \leq \frac{1-B_{\beta}}{2} \int_{0}^{N} x^{\alpha+\beta} f_{N}(x) d x+C_{\beta} \int_{0}^{N} x f_{N}(x) d x \\
& \leq \frac{1-B_{\beta}}{2} \int_{0}^{N} x^{\alpha+\beta} f_{N}(x) d x+C_{\beta} .
\end{aligned}
$$

Combining (27) and the previous estimate yields (25).

We next multiply $(12)$ by $x^{2}$, integrate over $(0, N)$ and use the non-negativity of $f_{N}$, $a$ and $b$ to obtain

$$
N f_{N}(N)-2 \int_{0}^{N} f_{N}(x) d x \geq-\int_{0}^{N} x^{2+\alpha} f_{N}(x) d x .
$$

It then follows from (24), (25) and the Hölder inequality that

$$
2 \int_{0}^{N} f_{N}(x) d x \leq \frac{C_{3}}{N}+C_{3}^{(\alpha+1) /(\alpha+2)},
$$

which completes the proof of Lemma 4.

REMARK 5. We point out here that Lemma 4 illustrates the opposite effects of the fragmentation and diffusion on the size distribution. Indeed, on the one hand, the bound (25) on moments of $f_{N}$ of high order results from the assumptions on the fragmentation and prevents the diffusion to expand the size distribution to the right. On the other hand, the $L^{1}$-bound (26) follows from the diffusive term and shows that diffusion prevents the concentration of the size distribution near $x=0$.

Lemma 6. There is a constant $C$ depending only on $\alpha$ such that

$$
\left\|f_{N}\right\|_{W^{1, \infty}(0, N)} \leq C \text {. }
$$


Proof. It follows from (12) and (13) that, for $x \in(0, N)$,

$$
\begin{aligned}
\partial_{x} f_{N}(x) & =\partial_{x} f_{N}(N)-\int_{x}^{N} \partial_{x}^{2} f_{N}\left(x^{\prime}\right) d x^{\prime} \\
& =\frac{f_{N}(N)}{N}-\int_{x}^{N} a\left(x^{\prime}\right) f_{N}\left(x^{\prime}\right) d x^{\prime}+\int_{x}^{N} \int_{x^{\prime}}^{N} a\left(x_{*}\right) b\left(x^{\prime}, x_{*}\right) f_{N}\left(x_{*}\right) d x_{*} d x^{\prime} .
\end{aligned}
$$

Since $\alpha \geq 0$, we infer from (8), (25), (26) and the Hölder inequality that

$$
\begin{aligned}
\left|\partial_{x} f_{N}(x)\right| & =\frac{f_{N}(N)}{N}+\int_{0}^{N} a(x) f_{N}(x) d x+\int_{0}^{N} a\left(x_{*}\right) f_{N}\left(x_{*}\right) \int_{0}^{x_{*}} b\left(x, x_{*}\right) d x d x_{*} \\
& \leq \frac{C_{3}}{N^{3}}+\left(1+B_{0}\right) \int_{0}^{N} x^{\alpha} f_{N}(x) d x \\
& \leq C_{3}+\left(1+B_{0}\right)\left\|f_{N}\right\|_{L^{1}(0, N)}^{3 /(\alpha+3)}\left(\int_{0}^{N} x^{\alpha+3} f_{N}(x) d x\right)^{\alpha /(\alpha+3)}, \\
\left|\partial_{x} f_{N}(x)\right| & \leq C .
\end{aligned}
$$

Now, since

$$
0 \leq f_{N}(x)=\int_{0}^{x} \partial_{x} f_{N}\left(x_{*}\right) d x_{*}
$$

by (13), Lemma 6 readily follows from (29).

Proof of Theorem 1. It readily follows from (25), (28) and the Vitali theorem that $\left(f_{N}\right)$ is relatively compact in $L^{1}\left(0, \infty ; x^{\beta} d x\right)$ for each $\beta \geq 0$, while (28) and the Ascoli theorem entail the relative compactness of $\left(f_{N}\right)$ in $\mathcal{C}([0, R])$ for each $R>0$. Consequently, there are a subsequence of $\left(f_{N}\right)$ (not relabeled) and

$$
f \in \mathcal{C}([0, \infty)) \cap \bigcap_{\beta \geq 0} L^{1}\left(0, \infty ; x^{\beta} d x\right)
$$

such that $\left(f_{N}\right)$ converges towards $f$ uniformly on compact subsets of $[0, \infty)$ and

$$
\lim _{N \rightarrow \infty} \int_{0}^{\infty} x^{\beta}\left|f_{N}(x)-f(x)\right| d x=0
$$

for each $\beta \geq 0$. In particular, $f$ is non-negative. Also, (30) allows us to let $N \rightarrow \infty$ in (24) and obtain that

$$
\int_{0}^{\infty} x f(x) d x=1 .
$$

In addition, we may pass to the limit as $N \rightarrow \infty$ in (12) and (13) and use classical arguments to conclude that $f \in W^{2,1}(0, \infty) \cap W_{0}^{1,1}(0, \infty)$ is a solution to (3), (4).

Finally, owing to the integrability properties required in Theorem 1, the proof of the uniqueness part of Theorem 1 is similar to that of Proposition 3.

\section{References}

[1] J. Banasiak and W. Lamb, On the application of substochastic semigroup theory to fragmentation models with mass loss, J. Math. Anal. Appl. 284 (2003), 9-30. 
[2] J. Carr, Asymptotic behaviour of solutions to the coagulation-fragmentation equations. I. The strong fragmentation case, Proc. Roy. Soc. Edinburgh Sect. A 121 (1992), 231-244.

[3] A. Eibeck and W. Wagner, Approximative solution of the coagulation-fragmentation equation by stochastic particle systems, Stochastic Anal. Appl. 18 (2000), 921-948.

[4] M. H. Ernst and G. Szamel, Fragmentation kinetics, J. Phys. A 26 (1993), 6085-6091.

[5] M. Escobedo, Ph. Laurençot, S. Mischler and B. Perthame, Gelation and mass conservation in coagulation-fragmentation models, J. Differential Equations 195 (2003), 143-174.

[6] J. Ferkinghoff-Borg, M. H. Jensen, J. Mathiesen, P. Olesen and K. Sneppen, Competition between diffusion and fragmentation: an important evolutionary process of nature, Phys. Rev. Lett. 91, 266103 (2003).

[7] A. F. Filippov, On the distribution of the sizes of particles which undergo splitting, Theory Probab. Appl. 6 (1961), 275-294.

[8] B. Haas, Loss of mass in deterministic and random fragmentations, Stochastic Process. Appl. 106 (2003), 245-277.

[9] E. D. McGrady and R. M. Ziff, "Shattering" transition in fragmentation, Phys. Rev. Lett. 58 (1987), 892-895.

[10] D. J. McLaughlin, W. Lamb and A. C. McBride, A semigroup approach to fragmentation models, SIAM J. Math. Anal. 28 (1997), 1158-1172.

[11] Z. A. Melzak, A scalar transport equation, Trans. Amer. Math. Soc. 85 (1957), 547-560.

[12] R. M. Ziff and E. D. McGrady, The kinetics of cluster fragmentation and depolymerisation, J. Phys. A 18 (1985), 3027-3037. 